\title{
Core Structure in Roselipins Essential for Eliciting Inhibitory Activity against
}

\section{Diacylglycerol Acyltransferase}

\author{
Hiroshi Tomoda*, Noriko Tabata, Yukako Ohyama and Satoshi ŌMUra* \\ Kitasato Institute for Life Sciences \& Graduate School of Infection Control Sciences, \\ Kitasato University, and The Kitasato Institute \\ Shirokane, Minato-ku, Tokyo 108-8641, Japan
}

(Received for publication September 26, 2002)

\begin{abstract}
Fungal roselipins, discovered as inhibitors of diacylglycerol acyltransferase (DGAT), consist of three parts; highly methylated C20 fatty acid, mannose and arabinitol. Demannosyl and/or dearabinitoyl roselipins were prepared chemically or enzymatically. Demannnosyl roselipins conserved the DGAT inhibitory activity, but the others lost the activity, indicating that the arabinitoyl fatty acid core is essential for eliciting the activity.
\end{abstract}

Roselipins 1A, 1B, 2A and 2B were isolated as inhibitors of diacylglycerol acyltransferase (DGAT) from the culture broth of Gliocladium roseum KF-1040 $0^{1,2)}$. They consist of three common parts; 2,4,6,8,10,12,14,16,18-nonamethyl5,9,13-trihydroxy-2E,6E,10E-icosatrienoic acid, D-mannose glycosylated at $13-\mathrm{OH}$ and $\mathrm{D}$-arabinitol esterified at 1$\mathrm{COOH}$. The roselipin A and B groups are stereoisomers at the arabinitol moiety, which esterifies the fatty acid from the different hydroxy terminals, and the roselipin 2 group is the derivative acetylated at $6-\mathrm{OH}$ of mannose in the roselipin 1 group $^{3)}$. All roselipins showed DGAT inhibitory activity to similar extents and roselipins $1 \mathrm{~A}$ and $1 \mathrm{~B}$ showed anti-Aspergillus niger activity ${ }^{2}$. It is intriguing to test which part(s) in the structures is involved in the biological activities.

In this paper, demannosyl and/or dearabinitoyl roselipins are prepared chemically or enzymatically, and we show that demannosyl roselipin is the core structure for DGAT inhibition.

\section{Experimental}

General Experimental Procedures

UV spectra were recorded on a Shimadzu UV-200S spectrophotometer. IR spectra were recorded on a Horiba FT-210 infrared spectrometer. Optical rotations were obtained with a JASCO DIP-370 digital polarimeter. Melting points were measured with a Yanaco micro melting point apparatus. EI-MS spectra were recorded on a JEOL JMS-D 100 mass spectrometer at $20 \mathrm{eV}$. FAB-MS spectra were recorded on a JMS-DX300 mass spectrometer. The various NMR spectra were obtained on a Varian XL-400 spectrometer. HPLC was carried out using the JASCO (TRI ROTAR V) system with a YMC-pack column (D-ODS, $20 \times 250 \mathrm{~mm}$ ) (Yamamura Chem. Co.).

\section{Roselipins}

Roselipins 1A, 1B, 2A and 2B were purified from the culture broth of Gliocladium roseum KF-1040 as reported previously ${ }^{1,2)}$.

Preparation of Roselipin Derivatives R-3A and R-3B

Roselipin 1A $(8.2 \mathrm{mg})$ was dissolved in $0.05 \mathrm{M}$ citrate buffer $(\mathrm{pH} 4.5,10 \mathrm{ml})$ and $10 \mathrm{mg} / \mathrm{ml}$ sodium taurodeoxycholate $(100 \mu \mathrm{l})$. Then, $\beta$-mannosidase (snail origin, Sigma) was added to the solution on day 0 (20 units) and day 8 ( 10 units), and the mixture was incubated at $37^{\circ} \mathrm{C}$ for 30 days. The degraded product was extracted with ethyl acetate and the derivative R-3A was purified by HPLC (solvent, $80 \% \mathrm{CH}_{3} \mathrm{CN}$; flow rate, $6 \mathrm{ml} /$ minute; detection, $\mathrm{UV}$ at $220 \mathrm{~nm}$ ). R-3A was eluted as a peak with a retention time of 35 minutes. The peak was collected and the fraction was concentrated to give pure R-3A ( $3.2 \mathrm{mg})$ as a colorless

* Corresponding author: tomoda@lisci.kitasato-u.ac.jp or omura-s@kitasato.or.jp 
oil.

Roselipin 1B $(3.0 \mathrm{mg})$ was dissolved in $0.05 \mathrm{M}$ citrate buffer $(\mathrm{pH} 4.5,10 \mathrm{ml})$ and $10 \mathrm{mg} / \mathrm{ml}$ sodium taurodeoxycholate $(100 \mu \mathrm{l})$. To the solution was added $\beta$-mannosidase on day 0 (20 units) and day 10 (5 units), and the mixture was incubated at $37^{\circ} \mathrm{C}$ for 30 days. The degraded product $\mathrm{R}-3 \mathrm{~B}$ was purified in a similar way. Under the same HPLC conditions, $\mathrm{R}-3 \mathrm{~B}$ was eluted as a peak with a retention time of 35 minutes. The peak was collected and the fraction was concentrated to give pure R-3B (1.0 mg) as a colorless oil.

\section{Preparation of Roselipin Derivative R-4}

A mixture of roselipins $1 \mathrm{~A}$ and $1 \mathrm{~B}$ (50 mg) was dissolved in $\mathrm{CH}_{3} \mathrm{OH}(1 \mathrm{ml})$, and was permitted to stand at room temperature for 2 days. After evaporation, derivative $\mathrm{R}-4$ was purified by HPLC (solvent, $80 \% \mathrm{CH}_{3} \mathrm{CN}$; flow rate, $6 \mathrm{ml} /$ minute; detection, $\mathrm{UV}$ at $220 \mathrm{~nm}$ ). R-4 was eluted as a peak with a retention time of 64 minutes. The peak was collected and the fraction was concentrated to give pure $\mathrm{R}-4$ (26 mg) as a colorless oil.
Preparation of Roselipin Derivative R-5

A mixture of roselipins $(20 \mathrm{mg})$ was dissolved in $2 \mathrm{~N}$ $\mathrm{HCl}-\mathrm{CH}_{3} \mathrm{OH}(1 \mathrm{ml})$, which was heated at $80^{\circ} \mathrm{C}$ for 12 hours. After evaporation, derivative R-5 was purified by HPLC (solvent, $100 \% \mathrm{CH}_{3} \mathrm{CN}$; flow rate, $6 \mathrm{ml} / \mathrm{minute}$; detection, UV at $220 \mathrm{~nm}$ ). R-5 was eluted as a peak with a retention time of 58 minutes. The peak was collected and the fraction was concentrated to give pure R-5 (1.4 mg) as a colorless oil.

\section{DGAT Activity}

DGAT activity was assayed in an enzyme assay using rat liver microsomes ${ }^{2,4)}$ and in a cell assay using Raji cells as reported previously ${ }^{5)}$.

\section{Antimicrobial Activity}

Antimicrobial activity was tested using paper disks (6 mm, ADVANTEC). Bacteria were grown on MüllerHinton agar medium (Difco), and fungi and yeasts were grown on potato broth agar medium. Antimicrobial activity was observed after a 24-hour incubation at $37^{\circ} \mathrm{C}$ for

Table 1. Physico-chemical properties of roselipin derivatives R-3A, R-3B, R-4 and R-5.

\begin{tabular}{|c|c|c|c|c|}
\hline & \multicolumn{4}{|c|}{ Derivative } \\
\hline & R-3A & R-3B & $\mathrm{R}-4$ & $\mathrm{R}-5$ \\
\hline Appearance & Colorless oil & Colorless oil & Colorless oil & Colorless oil \\
\hline $\begin{array}{l}\text { Molecular formula } \\
\text { Molecular weight } \\
\text { FAB-MS }(m / z)\end{array}$ & $\begin{array}{l}\mathrm{C}_{34} \mathrm{H}_{62} \mathrm{O}_{9} \\
614\end{array}$ & $\begin{array}{l}\mathrm{C}_{34} \mathrm{H}_{62} \mathrm{O}_{9} \\
614\end{array}$ & $\begin{array}{l}\mathrm{C}_{36} \mathrm{H}_{64} \mathrm{O}_{10} \\
656\end{array}$ & $\begin{array}{l}\mathrm{C}_{31} \mathrm{H}_{56} \mathrm{O}_{5} \\
508\end{array}$ \\
\hline Positive & $\begin{array}{l}615[\mathrm{M}+\mathrm{H}]^{+} \\
637[\mathrm{M}+\mathrm{Na}]^{+}\end{array}$ & $\begin{array}{l}615[\mathrm{M}+\mathrm{H}]^{+} \\
637[\mathrm{M}+\mathrm{Na}]^{+}\end{array}$ & $679[\mathrm{M}+\mathrm{Na}]^{+}$ & $531[\mathrm{M}+\mathrm{Na}]^{+}$ \\
\hline Negative & $613[\mathrm{M}-\mathrm{H}]^{-}$ & $613[\mathrm{M}-\mathrm{H}]^{-}$ & $655[\mathrm{M}-\mathrm{H}]^{-}$ & $507[\mathrm{M}-\mathrm{H}]^{-}$ \\
\hline HRFAB-MS ( $m / z)$ & $\begin{array}{l}\mathrm{C}_{34} \mathrm{H}_{62} \mathrm{O}_{9} \mathrm{Na} \\
{[\mathrm{M}+\mathrm{Na}]^{+}}\end{array}$ & $\begin{array}{l}\mathrm{C}_{34} \mathrm{H}_{62} \mathrm{O}_{9} \mathrm{Na} \\
{[\mathrm{M}+\mathrm{Na}]^{+}}\end{array}$ & $\begin{array}{l}\mathrm{C}_{36} \mathrm{H}_{64} \mathrm{O}_{10} \mathrm{Na} \\
{[\mathrm{M}+\mathrm{Na}]^{+}}\end{array}$ & $\begin{array}{l}\mathrm{C}_{31} \mathrm{H}_{56} \mathrm{O}_{5} \mathrm{Na} \\
{[\mathrm{M}+\mathrm{Na}]^{+}}\end{array}$ \\
\hline $\begin{array}{l}\text { Calcd: } \\
\text { Found: }\end{array}$ & $\begin{array}{l}637.4291 \\
637.4283\end{array}$ & $\begin{array}{l}637.4291 \\
637.4273\end{array}$ & $\begin{array}{l}679.4397 \\
679.4391\end{array}$ & $\begin{array}{l}531.4025 \\
531.4030\end{array}$ \\
\hline$[\alpha]_{\mathrm{D}}^{24}$ & $\begin{array}{l}+18^{\circ} \\
(c 0.1, \mathrm{MeOH})\end{array}$ & $\begin{array}{l}+10^{\circ} \\
(c 0.054, \mathrm{MeOH})\end{array}$ & $\begin{array}{l}+12^{\circ} \\
(c 0.1, \mathrm{MeOH})\end{array}$ & $\begin{array}{l}+14^{\circ} \\
(c 0.028, \mathrm{MeOH})\end{array}$ \\
\hline $\mathrm{UV} \lambda_{\max }^{\mathrm{CH}_{3} \mathrm{OH}} \mathrm{nm}(\varepsilon)$ & $\begin{array}{l}202(19,000) \\
222(14,100)\end{array}$ & $\begin{array}{l}202(30,100) \\
222(22,700)\end{array}$ & $\begin{array}{l}202(17,700) \\
221(13,300)\end{array}$ & $\begin{array}{l}206(62,900) \\
216(59,900)\end{array}$ \\
\hline $\operatorname{IR} v_{\max }^{\mathrm{KBr}}\left(\mathrm{cm}^{-1}\right)$ & $\begin{array}{l}3421,2960,2926, \\
2873,2854,1714, \\
1647,1583,1456, \\
1377,1271,1227, \\
1078,1018\end{array}$ & $\begin{array}{l}3430,2960,2929 \\
2873,2854,1701 \\
1632,1583,1458 \\
1385,1273,1228, \\
1042,1018\end{array}$ & $\begin{array}{l}3430,2962,2926, \\
2875,1714,1641, \\
1458,1379,1269, \\
1227,1124,1072, \\
1026\end{array}$ & $\begin{array}{l}3434,2962,2927 \\
2873,1718,1655 \\
1458,1377,1269 \\
1234,1128,1099 \\
1028\end{array}$ \\
\hline $\begin{array}{l}\text { Solubility } \\
\text { Soluble: }\end{array}$ & $\begin{array}{l}\mathrm{CH}_{3} \mathrm{OH}, \mathrm{CHCl}_{3}, \\
\mathrm{CH}_{3} \mathrm{CN}, \text { acetone, } \\
\mathrm{C}_{2} \mathrm{H}_{5} \mathrm{OH} \text {, } \\
\text { ethyl acetate }\end{array}$ & $\begin{array}{l}\mathrm{CH}_{3} \mathrm{OH}, \mathrm{CHCl}_{3}, \\
\mathrm{CH}_{3} \mathrm{CN}, \text { acetone, } \\
\mathrm{C}_{2} \mathrm{H}_{5} \mathrm{OH} \text {, } \\
\text { ethyl acetate }\end{array}$ & $\begin{array}{l}\mathrm{CH}_{3} \mathrm{OH}, \mathrm{CHCl}_{3}, \\
\mathrm{CH}_{3} \mathrm{CN}, \text { acetone, } \\
\mathrm{C}_{2} \mathrm{H}_{5} \mathrm{OH}, \\
\text { ethyl acetate }\end{array}$ & $\begin{array}{l}\mathrm{CH}_{3} \mathrm{OH}, \mathrm{CHCl}_{3} \text {, } \\
\mathrm{CH}_{3} \mathrm{CN} \text {, acetone, } \\
\mathrm{C}_{2} \mathrm{H}_{5} \mathrm{OH} \text {, } \\
\text { ethyl acetate }\end{array}$ \\
\hline $\begin{array}{l}\text { Insoluble: } \\
\text { Color reaction }\end{array}$ & $\mathrm{H}_{2} \mathrm{O}, n$-hexane & $\mathrm{H}_{2} \mathrm{O}, n$-hexane & $\mathrm{H}_{2} \mathrm{O}, n$-hexane & $\mathrm{H}_{2} \mathrm{O}, \boldsymbol{n}$-hexane \\
\hline $\begin{array}{l}\text { Positive: } \\
\text { Negative: }\end{array}$ & $\begin{array}{l}50 \% \mathrm{H}_{2} \mathrm{SO}_{4} \\
\text { Ninhydrin reagent }\end{array}$ & $\begin{array}{l}50 \% \mathrm{H}_{2} \mathrm{SO}_{4} \\
\text { Ninhydrin reagent }\end{array}$ & $\begin{array}{l}50 \% \mathrm{H}_{2} \mathrm{SO}_{4} \\
\text { Ninhydrin reagent }\end{array}$ & $\begin{array}{l}50 \% \mathrm{H}_{2} \mathrm{SO}_{4} \\
\text { Ninhydrin reagent }\end{array}$ \\
\hline
\end{tabular}


bacteria and after a 48 -hour incubation at $27^{\circ} \mathrm{C}$ for fungi and yeasts.

\section{Results}

Structures of Roselipin Derivatives

Physico-chemical properties of roselipin derivatives are summarized in Table 1. In comparison with those of parent roselipins, all the signals of the derivatives observed by NMR experiments were completely assigned as shown in Tables 2 and 3. Eventually, derivative R-3A is demannnosyl roselipin $\mathrm{A}, \mathrm{R}-3 \mathrm{~B}$ is demannnosyl roselipin $\mathrm{B}, \mathrm{R}-4$ is dearabinytoyl roselipin 1 methyl ester, and $\mathrm{R}-5$ is demannnosyl-dearabinitoyl-5-O-methylroselipin methyl ester (Fig. 1). The elucidated structures were definitely supported by the physico-chemical properties.

\section{DGAT Inhibitory Activity}

Parent roselipins showed DGAT inhibition in the in vitro enzymatic assay with $\mathrm{IC}_{50}$ values of $15 \sim 22 \mu \mathrm{M}$. As listed in Table 4, derivatives R-3A and R-3B maintained the inhibitory activity ( $\mathrm{IC}_{50}$ values; 60 and $33 \mu \mathrm{M}$, respectively), although

Table 2. ${ }^{1} \mathrm{H}$ and ${ }^{13} \mathrm{C}$ NMR chemical shifts of roselipin derivatives R-3A and R-3B.

\begin{tabular}{|c|c|c|c|c|c|}
\hline \multirow{2}{*}{$\begin{array}{l}\text { Carbon } \\
\text { No. }\end{array}$} & \multicolumn{3}{|r|}{ Derivative R-3A } & \multicolumn{2}{|r|}{ Derivative R-3B } \\
\hline & $\begin{array}{l}{ }^{13} \mathrm{C} \text { chemi } \\
\text { shifts (ppn }\end{array}$ & & $\begin{array}{l}{ }^{1} \mathrm{H} \text { chemical } \\
\text { shifts }(\mathrm{ppm})^{\mathrm{b}}\end{array}$ & $\begin{array}{l}{ }^{13} \mathrm{C} \text { chemical } \\
\text { shifts }(\mathrm{ppm})^{\mathrm{a}}\end{array}$ & $\begin{array}{l}{ }^{1} \mathrm{H} \text { chemical } \\
\text { shifts }(\mathrm{ppm})^{\mathrm{b}}\end{array}$ \\
\hline C-1 & 170.05 & & & 169.82 & \\
\hline $\mathrm{C}-2$ & 128.81 & & & 128.73 & \\
\hline C-3 & 147.57 & 6.80 & $(1 \mathrm{H}, \mathrm{dd}, J=10.0,1.5 \mathrm{~Hz})$ & 147.71 & $6.78(1 \mathrm{H}, \mathrm{dd}, J=10.0,1.5 \mathrm{~Hz})$ \\
\hline C-4 & 38.02 & 2.73 & $(1 \mathrm{H}, \mathrm{m})$ & 38.07 & $2.75(1 \mathrm{H}, \mathrm{m})$ \\
\hline C-5 & 83.71 & 3.82 & $(1 \mathrm{H}, \mathrm{d}, J=8.5 \mathrm{~Hz})$ & 83.72 & $3.82(1 \mathrm{H}, \mathrm{d}, J=9.0 \mathrm{~Hz})$ \\
\hline C-6 & 137.14 & & & 137.20 & \\
\hline $\mathrm{C}-7$ & 134.30 & 5.33 & $(1 \mathrm{H}, \mathrm{dd}, J=9.0,1.0 \mathrm{~Hz})$ & 134.30 & $5.33(1 \mathrm{H}, \mathrm{dd}, J=9.0,1.0 \mathrm{~Hz})$ \\
\hline $\mathrm{C}-8$ & 37.08 & 2.62 & $(1 \mathrm{H}, \mathrm{m})$ & 37.12 & $2.63(1 \mathrm{H}, \mathrm{m})$ \\
\hline C-9 & 84.33 & 3.72 & $(1 \mathrm{H}, \mathrm{d}, J=9.5 \mathrm{~Hz})$ & 84.31 & $3.72(1 \mathrm{H}, \mathrm{d}, J=9.5 \mathrm{~Hz})$ \\
\hline C-10 & 134.79 & & & 134.84 & \\
\hline C-11 & 134.69 & 5.56 & $(1 \mathrm{H}, \mathrm{dd}, J=9.5,1.0 \mathrm{~Hz})$ & 134.67 & $5.57(1 \mathrm{H}, \mathrm{dd}, J=9.5,1.5 \mathrm{~Hz})$ \\
\hline $\mathrm{C}-12$ & 36.20 & 2.75 & $(1 \mathrm{H}, \mathrm{m})$ & 36.24 & $2.76(1 \mathrm{H}, \mathrm{m})$ \\
\hline$C-13$ & 87.31 & 3.49 & $(1 \mathrm{H}, \mathrm{dd}, J=7.0,3.5 \mathrm{~Hz})$ & 87.33 & $3.49(1 \mathrm{H}, \mathrm{dd}, J=7.0,3.5 \mathrm{~Hz})$ \\
\hline C-14 & 34.27 & 1.87 & $(1 \mathrm{H}, \mathrm{m})$ & 34.29 & $1.87(1 \mathrm{H}, \mathrm{m})$ \\
\hline C-15 & 43.93 & $\begin{array}{l}0.95 \\
1.37\end{array}$ & $\begin{array}{l}(1 \mathrm{H}, \mathrm{m}) \\
(1 \mathrm{H}, \mathrm{m})\end{array}$ & 43.97 & $\begin{array}{l}1.02(1 \mathrm{H}, \mathrm{m}) \\
1.42(1 \mathrm{H}, \mathrm{m})\end{array}$ \\
\hline C-16 & 28.85 & 1.62 & $(1 \mathrm{H}, \mathrm{m})$ & 28.90 & $1.62(1 \mathrm{H}, \mathrm{m})$ \\
\hline $\mathrm{C}-17$ & 46.04 & $\begin{array}{l}0.88 \\
1.24\end{array}$ & $\begin{array}{l}(1 \mathrm{H}, \mathrm{m}) \\
(1 \mathrm{H}, \mathrm{m})\end{array}$ & 46.07 & $\begin{array}{l}0.94(1 \mathrm{H}, \mathrm{m}) \\
1.27(1 \mathrm{H}, \mathrm{m})\end{array}$ \\
\hline C-18 & 32.91 & 1.45 & $(1 \mathrm{H}, \mathrm{m})$ & 32.94 & $1.45(1 \mathrm{H}, \mathrm{m})$ \\
\hline C-19 & 29.86 & $\begin{array}{l}1.08 \\
1.42\end{array}$ & $(1 \mathrm{H}, \mathrm{m})$ & 29.89 & $\begin{array}{l}1.22(1 \mathrm{H}, \mathrm{m}) \\
1.45(1 \mathrm{H}, \mathrm{m})\end{array}$ \\
\hline $\mathrm{C}-20$ & 11.53 & 0.89 & $(3 \mathrm{H}, \mathrm{t}, J=7.0 \mathrm{~Hz})$ & 11.52 & $0.89(3 \mathrm{H}, \mathrm{t}, J=6.5 \mathrm{~Hz})$ \\
\hline$C-21$ & 12.88 & 1.90 & $(3 \mathrm{H}, \mathrm{d}, J=1.5 \mathrm{~Hz})$ & 12.87 & $1.89(3 \mathrm{H}, \mathrm{d}, J=1.5 \mathrm{~Hz})$ \\
\hline $\mathrm{C}-22$ & 16.81 & 0.86 & $(3 \mathrm{H}, \mathrm{d}, J=7.0 \mathrm{~Hz})$ & 16.82 & $0.85(3 \mathrm{H}, \mathrm{d}, J=7.0 \mathrm{~Hz})$ \\
\hline C-23 & 11.36 & 1.68 & $(3 \mathrm{H}, \mathrm{d}, J=1.0 \mathrm{~Hz})$ & $11.36^{\mathrm{d}}$ & $1.67(3 \mathrm{H}, \mathrm{d}, J=1.0 \mathrm{~Hz})$ \\
\hline $\mathrm{C}-24$ & 17.82 & 0.78 & $(3 \mathrm{H}, \mathrm{d}, J=7.0 \mathrm{~Hz})$ & 17.82 & $0.78(3 \mathrm{H}, \mathrm{d}, J=7.0 \mathrm{~Hz})$ \\
\hline$C-25$ & 11.32 & 1.64 & $(3 \mathrm{H}, \mathrm{d}, J=1.0 \mathrm{~Hz})$ & $11.36^{\mathrm{d})}$ & $1.64(3 \mathrm{H}, \mathrm{d}, J=1.5 \mathrm{~Hz})$ \\
\hline C-26 & 18.63 & 0.99 & $(3 \mathrm{H}, \mathrm{d}, J=7.0 \mathrm{~Hz})$ & 18.63 & $0.99(3 \mathrm{H}, \mathrm{d}, J=7.0 \mathrm{~Hz})$ \\
\hline C-27 & 15.56 & 0.94 & $(3 \mathrm{H}, \mathrm{d}, J=7.0 \mathrm{~Hz})$ & 15.58 & $0.95(3 \mathrm{H}, \mathrm{d}, J=7.0 \mathrm{~Hz})$ \\
\hline $\mathrm{C}-28$ & 21.34 & 0.90 & $(3 \mathrm{H}, \mathrm{d}, J=6.5 \mathrm{~Hz})$ & 21.35 & $0.90(3 \mathrm{H}, \mathrm{d}, J=7.0 \mathrm{~Hz})$ \\
\hline $\mathrm{C}-29$ & 20.73 & 0.90 & $(3 \mathrm{H}, \mathrm{d}, J=6.5 \mathrm{~Hz})$ & 20.73 & $0.90(3 \mathrm{H}, \mathrm{d}, J=7.0 \mathrm{~Hz})$ \\
\hline C-1' & 67.88 & $\begin{array}{l}4.25 \\
4.41\end{array}$ & $\begin{array}{l}(1 \mathrm{H}, \mathrm{dd}, J=12.0,6.5 \mathrm{~Hz}) \\
(1 \mathrm{H}, \mathrm{dd}, J=12.0,3.0 \mathrm{~Hz})\end{array}$ & 67.16 & $\begin{array}{l}4.20(1 \mathrm{H}, \mathrm{dd}, J=11.0,5.5 \mathrm{~Hz}) \\
4.27(1 \mathrm{H}, \mathrm{dd}, J=11.0,7.0 \mathrm{~Hz})\end{array}$ \\
\hline C-2' & 70.63 & 3.94 & $(1 \mathrm{H}, \mathrm{ddd}, J=9.0,6.5,3.0 \mathrm{~Hz})$ & 69.38 & $4.14(1 \mathrm{H}, \mathrm{ddd}, J=7.0,5.5,2.0 \mathrm{~Hz})$ \\
\hline C-3' & 71.93 & 3.58 & $1 \mathrm{H}, \mathrm{dd}, J=9.0,2.0 \mathrm{~Hz})$ & 72.30 & $3.53(1 \mathrm{H}, \mathrm{dd}, J=8.0,2.0 \mathrm{~Hz})$ \\
\hline C-4' & 71.64 & 3.92 & $(1 \mathrm{H}, \mathrm{ddd}, J=6.5,6.5,2.0 \mathrm{~Hz})$ & 72.71 & $3.73(1 \mathrm{H}, \mathrm{ddd}, J=8.0,6.0,3.5 \mathrm{~Hz})$ \\
\hline C-5' & 64.80 & 3.65 & $(2 \mathrm{H}, \mathrm{ddd}, J=7.0,6.5,6.5 \mathrm{~Hz})$ & 65.03 & $\begin{array}{l}3.64(1 \mathrm{H}, \mathrm{dd}, J=11.0,6.0 \mathrm{~Hz}) \\
3.81(1 \mathrm{H}, \mathrm{dd}, J=11.0,3.5 \mathrm{~Hz})\end{array}$ \\
\hline
\end{tabular}

a) Chemical shifts are shown with reference to $\mathrm{CD}_{3} \mathrm{OD}$ as $49.8 \mathrm{ppm}$. ${ }^{\text {b) }}$ Chemical shifts are shown with reference to $\mathrm{CD}_{3} \mathrm{OD}$ as $3.30 \mathrm{ppm}{ }^{\text {c) }}$ Signal was observed as a double doublet with ${ }^{2} J_{\mathrm{CH}}=6.8 \mathrm{~Hz}$. ${ }^{\text {d) }} \mathrm{The}$ signals were observed as the same chemical shifts. 
Table 3. $\quad{ }^{1} \mathrm{H}$ and ${ }^{13} \mathrm{C}$ NMR chemical shifts of roselipin derivatives R-4 and R-5.

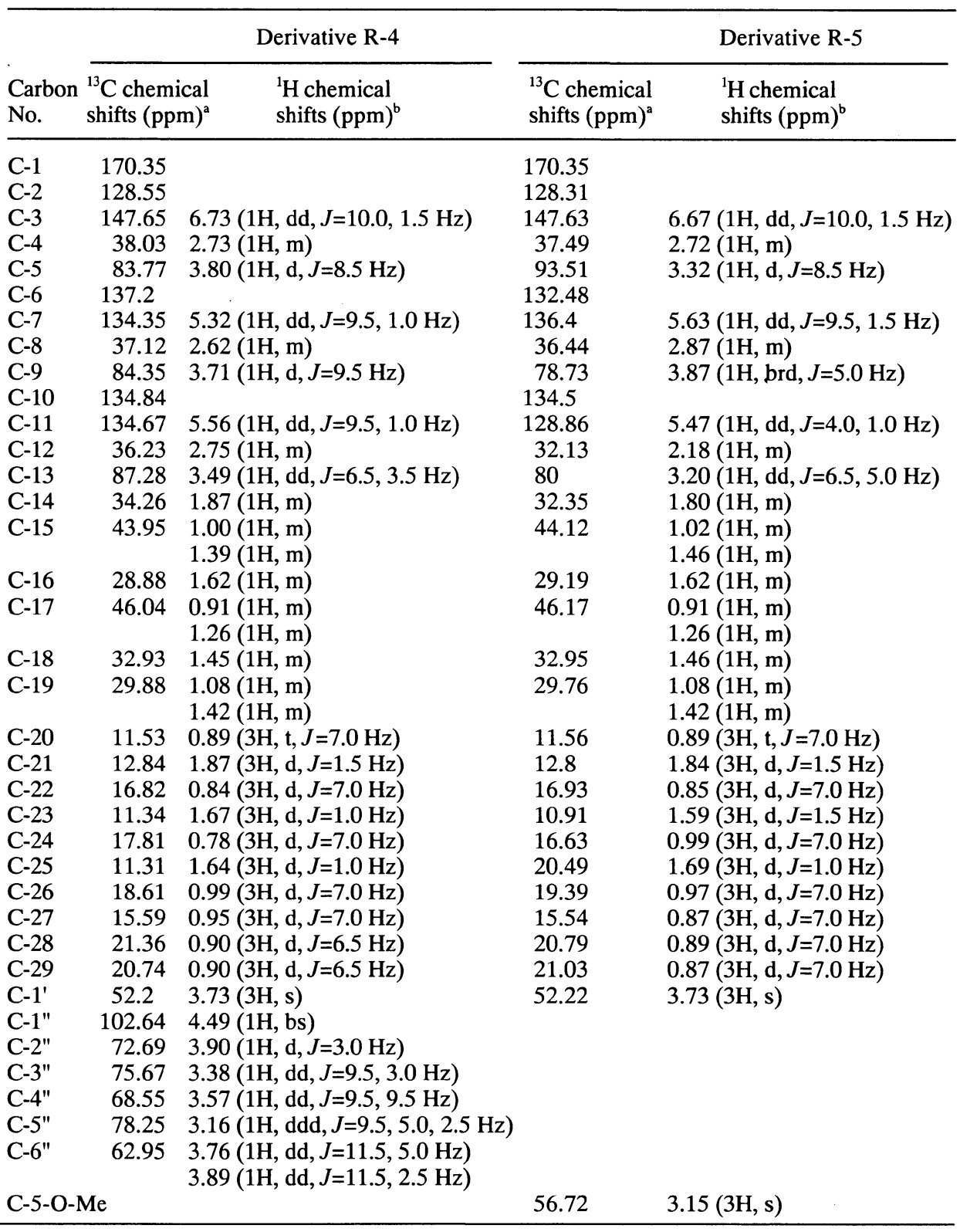

a) Chemical shifts are shown with reference to $\mathrm{CD}_{3} \mathrm{OD}$ as $49.8 \mathrm{ppm} .{ }^{\text {b) }}$ Chemical shifts are shown with reference to $\mathrm{CD}_{3} \mathrm{OD}$ as $3.30 \mathrm{ppm}$. ${ }^{\text {c) }}$ Signal was observed as a double doublet with ${ }^{2} J_{\mathrm{CH}}=6.8 \mathrm{H}$ d) The signals were observed as the same chemical shifts.

the potency slightly reduced. On the other hand, derivatives R-4 and R-5 completely lost the inhibitory activity.

In the cell assay using Raji cells (Table 4), derivatives R3A and R-3B inhibited triacylglycerol (TG) synthesis with $\mathrm{IC}_{50}$ values of 11 and $10 \mu \mathrm{M}$, respectively, showing more potent than parent roselipins in the cell assay. Derivatives R-4 and R-5 showed very weak inhibition as expected from the results of the in vitro assay.

\section{Antifungal Activities}

Almost no antibacterial activity of the four derivatives was observed at a concentration of $1.0 \mathrm{mg} / \mathrm{ml}(10 \mu \mathrm{g} / 6 \mathrm{~mm}$ disk) against the following microorganism; Bacillus subtilis, Mycobacterium smegmatis, Pseudomonas aeruginosa, Escherichia coli, Micrococcus luteus and Staphylococcus aureus. Regarding their antifungal and 
Fig. 1. Structures of roselipin derivatives.<smiles>CCC(C)CC(C)CC(C)C(O)C(C)C=C(C)C(O)C(C)C=C(C)C(O)C(C)C=C(C)C(=O)O</smiles>

\begin{tabular}{|c|c|c|c|}
\hline Roselipin derivatives & $\mathbf{R}_{1}$ & $\mathbf{R}_{2}$ & $\mathbf{R}_{\mathbf{3}}$ \\
\hline R-3A & $-H$ & $-\mathrm{H}$ & \\
\hline R-3B & $-\mathrm{H}$ & $-H$ & \\
\hline R-4 & & $-H$ & $-{ }_{-1}^{\prime} \mathrm{CH}_{3}$ \\
\hline R-5 & $-H$ & $-\mathrm{CH}_{3}$ & $-\mathrm{CH}_{3}$ \\
\hline
\end{tabular}

Table 4. DGAT inhibitory activity of roselipin derivatives R-3A, R-3B, R-4 and R-5.

\begin{tabular}{rcc}
\hline \multirow{2}{*}{ Compound } & \multicolumn{2}{c}{$\mathrm{IC}_{50}(\mu \mathrm{M})$} \\
\cline { 2 - 3 } & Enzyme assay & Cell assay \\
\hline Roselipin 1A & 17 & 39 \\
1B & 15 & 32 \\
2A & 22 & 24 \\
2B & 18 & 18 \\
Derivative R-3A & 60 & 11 \\
R-3B & 33 & 10 \\
R-4 & $>760$ & 200 \\
R-5 & $>960$ & 250 \\
\hline
\end{tabular}


antiyeast activities against Candida albicans, Saccharomyces cerevisiae, Pyricularia oryzae, Mucor racemosus and Aspergillus niger, derivatives R-3A and R-4 were active against Mucor racemosus (diameter of inhibition zone; 14 and $12 \mathrm{~mm}$, respectively) and Pyricularia oryzae (0 and $16 \mathrm{~mm}$, respectively) at 1.0 $\mathrm{mg} / \mathrm{ml}(10 \mu \mathrm{g} / 6 \mathrm{~mm}$ disk). They lost anti-A. niger activity, although their parent roselipins $1 \mathrm{~A}$ and $1 \mathrm{~B}$ showed the activity. Derivatives R-3B and R-5 showed no antifungal and antiyeast activities at the concentration. Difference in the antifungal activity between derivatives R-3A and R-3B may be that the binding form of the arabinitol to the fatty acid core is significant for antifungal activity. These results are not coincident with those in DGAT inhibition, probably due to its different mode of action.

\section{Discussion}

Although they are not good substrates of the enzyme, roselipins $1 \mathrm{~A}$ and $1 \mathrm{~B}$ were hydrolyzed gradually by snail $\beta$ mannnosidase to produce demannosylated derivatives R-3A and R-3B after a 30 day-incubation. The derivatives maintained the DGAT inhibitory activity, while derivatives R-4 and R-5 lost the activity completely (Table 4), indicating that the arabinitoyl fatty acid core is essential for eliciting DGAT inhibitory activity. It might be that the core structure is mimic to diacylglycerol, the substrate of DGAT enzyme. The results in the cell assay are almost comparable to those in the enzyme assay. Derivatives R-3A and R-3B are more potent in the cell assay than roselipins (Table 4), suggesting that the derivatives are more membranepermeable than roselipins.

Farese and coworkers identified a gene encoding a mammalian DGAT (DGAT-1) ${ }^{6}$. Its mRNA expression was detected in every mammalian tissue, and the highest expression levels were found in small intestine. These findings are consistent with a proposed role for DGAT in intestinal fat absorption. However, mRNA expression was relatively low in the livers of humans regardless of high DGAT activity, suggesting the existence of a second DGAT in livers. Recently, a second mammalian DGAT (DGAT-2) was cloned and characterized ${ }^{7}$. DGAT- 1 and -2 showed no homology, but similar characteristics. Therefore, selectivity of our DGAT inhibitors ${ }^{1,8 \sim 10)}$ in DGAT-1 and -2 inhibition remains to be studied, and they will become the center of researchers' attention ${ }^{11)}$. Further understanding DGAT at a molecular level will help us search for DGAT inhibitors, leading to potential approaches for treating hypertriglyceridemia or obesity in humans.
Acknowledgments

We express our thanks to Ms. N. SATO, School of Pharmaceutical Sciences, Kitasato University, for measurement of NMR spectra.

\section{References}

1) Ōmura, S.; H. Tomoda, Y. Ohyama, N. Tabata, T. Abe \& M. NAMIKOSHI: Roselipins, novel fungal metabolites having a highly methylated fatty acid modified with a mannose and an arabinitol. J. Antibiotics 52: 586 589, 1999

2) Tomoda, H.; Y. Ohyama, T. Abe, N. Tabata, M. NAMiKoshi, Y. YamaguChI, R. Masuma \& S. ŌMURA: Roselipins, inhibitors of diacylglycerol acyltransferase produced by Gliocladium roseum KF-1040. J. Antibiotics 52: 689 694, 1999

3) Tabata, N.; Y. Ohyama, H. Tomoda, T. AвE, M. NAMIKOSHI \& S. ŌMURA: Structure elucidation of roselipins, inhibitors of diacylglycerol acyltransferase produced by Gliocladium roseum KF-1040. J. Antibiotics 52: 815 826, 1999

4) MAYOREK, N. \& J. BAR-TANA: Inhibition of diacylglycerol acyltransferase by 2-bromooctanoate in cultured rat hepatocytes. J. Biol. Chem. 260: 6528 6532,1985

5) Tomoda, H.; K. Igarashi, J.-C. Cyong \& S. Ōmura: Evidence for an essential role of long chain acyl-CoA synthetase in animal cell proliferation.-Inhibition of long chain acyl-CoA synthetase by triacsins caused inhibition of Raji cell proliferation. J. Biol. Chem. 266: 4214 4219, 1991

6) Case, S.; S. J. Smith, Y.-W. Zheng, H. M. Myers, S. R. Lear, E. Sande, S. Novak, C. Collins, C. B. Welch, A. J. Lusis, S. K. ERICKSON \& R. V. FARESE Jr.: Identification of a gene encoding an acyl CoA: diacylglycerol acyltransferase, a key enzyme in triacylglycerol synthesis. Proc. Natl. Acad. Sci. USA 95: 13018 13023, 1998

7) Case, S.; S. J. Stone, P. Zhou, E. Yen, B. Tow, K. D. Lardizabal, T. Voelker \& R. V. Farese Jr.: Cloning of DGAT-2, a second mammalian diacylglycerol acyltransferase, and related family members. J. Biol. Chem. 276: $38870 \sim 38876,2001$

8) Tomoda, H.; M. Ito, N. Tabata, R. Masuma, Y. YAMAGUCHI \& S. ŌMURA: Amidepsines, inhibitors of diacylglycerol acyltransferase produced by Humicola sp. FO-2942 I. Production, isolation and biological properties. J. Antibiotics 48: 937 941, 1995

9) Tomoda, H.; Y. Yamaguchi, N. Tabata, T. Kobayashi, R. Masuma, H. Tanaka, \& S. Ōmura: Amidepsine E, an inhibitor of diacylglycerol acyltransferase produced by Humicola sp. FO-5969. J. Antibiotics 49: 929 931, 1996

10) Tabata, N.; M. Ito, H. Tomoda \& S. Ōmura: Xanthohumols, diacylglycerol acyltransferase inhibitors, from Humulus lupulus. Phytochemistry 46: 683 687, 1997

11) Buhman, K. K.; H. C. Chen \& R. V. Farese Jr.: The enzymes of neutral lipid synthesis. J. Biol. Chem. 276: 40369 40372, 2001 